PROCEEDINGS OF THE

AMERICAN MATHEMATICAL SOCIETY

Volume 135, Number 1, January 2007, Pages 1-4

S 0002-9939(06)08437-1

Article electronically published on August 16, 2006

\title{
A REMARK ON CLOSED NONCOMMUTATIVE SUBSPACES
}

\author{
E. S. LETZTER
}

(Communicated by Martin Lorenz)

\begin{abstract}
Given an abelian category with arbitrary products, arbitrary coproducts, and a generator, we show that the closed subspaces (in the sense of A. L. Rosenberg) are parameterized by a suitably defined poset of ideals in the generator. In particular, the collection of closed subspaces is itself a small poset.
\end{abstract}

\section{INTRODUCTION}

Following Rosenberg [3], Van den Bergh [8], and others (the reader is referred to [6] for an overview), the fundamental objects of study in noncommutative algebraic geometry are Grothendieck categories, interpreted as categories of sheaves on (not explicitly defined) noncommutative schemes. Key to this approach is the notion of a closed subspace, investigated in detail, for example, in [1, 3], 4], [5], [8. This brief note records an elementary - but apparently previously unnoticedobservation about closed subspaces, working in the slightly more general setting of an abelian category equipped with a generator and having arbitrary products and coproducts: We observe that there is a duality between the collection of closed subspaces and a suitably defined small poset of ideals within the generator. (After the fact, the poset structure does not depend on the choice of generator.) This duality generalizes Rosenberg's duality, in the case of module categories, between closed subspaces and two-sided ideals [3, III.6.4.1, p. 127]. A different generalization can be found in $[8, \S 3.4]$.

As a corollary to the duality we present below, it follows that the collection of closed subspaces is a small set. Also, the closed subspaces satisfy the descending chain condition if and only if the poset of ideals satisfies the ascending chain condition. Consequences of the descending chain condition for closed subspaces can be found, for example, in [1], 4].

Received by the editors May 25, 2005 and, in revised form, July 19, 2005.

2000 Mathematics Subject Classification. Primary 18E15; Secondary 14A22.

The author thanks the Department of Mathematics at the University of Pennsylvania for its hospitality; the research for this paper was undertaken while he was a visitor on sabbatical there. The author is grateful for support during this period from a Temple University Research and Study Leave Grant. This research was also supported in part by a grant from the National Security Agency.

(C)2006 American Mathematical Society Reverts to public domain 28 years from publication 


\section{Closed subspaces And the poset of ideals}

Our approach is heavily influenced by [3], 4], 8]. The reader is referred to [2], [7] for background information on abelian categories.

2.0 Notation and assumptions. (i) Throughout this note, $A$ will denote an abelian category with coproducts and products (both over arbitrary sets) and equipped with a generator $\Lambda$. Examples for $A$ include module categories over rings (always assumed to be associative and unital) and Grothendieck categories. To avoid set-theoretic difficulties, we will always view $A$ as large and sets as small.

(ii) Recall that subobjects, quotient objects, and subquotient objects (i.e., quotients of subobjects) refer to equivalence classes of objects (cf., e.g., [7, pp. 83-84]). We will abuse the notation slightly by referring to morphisms, coproducts, products, etc. of these equivalences classes (rather than of their representative objects).

2.1. Let $M$ be an object in $A$. Since $A$ has a generator, the collection $L(M)$ of subobjects of $M$ is a set (cf. [7, IV.6.6]) and is also a lattice under sums, intersections, and inclusions (as defined in [7, p. 88]). In particular, the collection of subquotients of $M$ is a set.

2.2. Following [4, §2], [8, §3.3] we will say that a full subcategory $C$ of $A$ is closed if $C$ is closed under products, coproducts, subquotients, and isomorphisms. In the terminology of [4, the closed subcategories are closed subspaces.

2.3. Let $C$ be a closed subcategory of $A$. The collection of kernels of all of the morphisms from $\Lambda$ into objects in $C$ is a set, and we will use $I_{\Lambda}(C)$, the ideal of $C$ in $\Lambda$, to denote the intersection of these kernels. Also, we will say that a subobject of $\Lambda$ is an ideal in $\Lambda$ if it is the ideal of some closed subcategory of $A$.

As of now we do not know of an equivalent, more intrinsic, general description of the ideals in $\Lambda$.

2.4. For rings, the preceding definition coincides with the standard theory: Let $R$ be a ring, and let $I$ be a (two-sided) ideal of $R$, defined in the usual ring-theoretic way. Let $\operatorname{Mod} R$ denote the category of left $R$-modules, with generator $R$, and let $C(I)$ denote the full subcategory of $\operatorname{Mod} R$ consisting of those left $R$-modules $M$ for which $I . M=0$. It is easy to see that $C(I)$ is a closed subcategory of Mod $R$. It is also easy to see, in the notation of section 2.3, that $I=I_{R}(C(I))$. In [3, III.6.4.1] A. L. Rosenberg proved that every closed subcategory of $\operatorname{Mod} R$ has the form $C(I)$ for some ideal $I$, and it follows that the ideals defined in (2.3) for the generator $R$ of $\operatorname{Mod} R$ are exactly the usual ring-theoretic ideals of $R$. Hence, there is a bijective duality between closed subcategories of $\operatorname{Mod} R$ and ideals of $R$. It is this duality that we seek to generalize below. A different generalization may be found in [8, $\S 3.4]$.

2.5 Proposition. Let $C$ be a closed subcategory of $A$, and set $I=I_{\Lambda}(C)$. Then $\Lambda / I$ is contained in $C$ and is a generator for $C$. Consequently, if $C$ and $C^{\prime}$ are distinct closed subcategories of $A$, then $I_{\Lambda}(C) \neq I_{\Lambda}\left(C^{\prime}\right)$.

Proof. Let $S$ be the set of all quotients of $\Lambda$ contained in $C$. Let $M \in C$ be the product of these quotients, and observe that $I$ is equal to the intersection of the kernels of the canonical quotient morphisms from $\Lambda$ into objects contained in $S$. Therefore, $I$ is the kernel of the resulting product morphism from $\Lambda$ into $M$, and so 
$\Lambda / I \in C$. Now note that every object in $C$ is an epimorphic image of a coproduct of isomorphic copies of $\Lambda$, since $\Lambda$ is a generator for $A$ (cf. [2, 2.8.2, p. 51]). In other words, every object in $C$ is a sum of images of $\Lambda$. But the set of images of $\Lambda$ in $C$ coincides with the set of images of $\Lambda / I$ in $C$, and so every module in $C$ is a sum of images of $\Lambda / I$. Consequently, every module in $C$ is an epimorphic image of a coproduct of ismorphic copies of $\Lambda / I$. Hence, $\Lambda / I$ is a generator for $C$ (again cf. [2, 2.8.2, p. 51]), and the proposition follows.

2.6 Corollary. The collection of closed subcategories of $A$ is a small set.

2.7. When $I$ is an ideal of $\Lambda$ we will use $C_{\Lambda}(I)$ to denote the unique closed subcategory of $A$ whose ideal in $\Lambda$ is $I$. We regard the set of ideals in $\Lambda$ as a poset with respect to inclusion. We also regard the set of closed subcategories of $A$ as a poset with respect to inclusion (i.e., $C \subseteq C^{\prime}$ when every object of $C$ is contained in $C^{\prime}$ ).

2.8 Lemma. Let $C$ and $C^{\prime}$ be closed subcategories of $A$ with respective ideals $I$ and $I^{\prime}$ of $\Lambda$. Then $C \subseteq C^{\prime}$ if and only if $I^{\prime} \subseteq I$.

Proof. It follows immediately from the definition of ideal that $I^{\prime} \subseteq I$ if $C \subseteq C^{\prime}$. Now suppose that $I^{\prime} \subseteq I$. Then $\Lambda / I$ is a quotient of $\Lambda / I^{\prime}$, and in particular, $\Lambda / I$ is contained in $C^{\prime}$. Since $\Lambda / I$ is a generator for $C$, it follows that $C \subseteq C^{\prime}$.

We can combine Proposition 2.5 and Lemma 2.8 to obtain the following.

2.9 Theorem. The function

$$
\{\text { Closed Subcategories of } A\} \stackrel{C \longmapsto I_{\Lambda}(C)}{\longrightarrow}\{\text { Ideals in } \Lambda\}
$$

is a poset isomorphism.

2.10. In view of Theorem 2.9 , we see, after the fact, that the poset of ideals in $\Lambda$, up to a poset isomorphism, does not depend on the choice of generator. As an application of this fact, we see that $A$ satisfies the descending chain condition for closed subcategories if and only if the poset of ideals satisfies the ascending chain condition. (Consequences of the descending chain condition for closed subcategories can be found, for example, in 11, 4].) As another application, when $\Lambda$ is projective, it then follows from section 2.4 and (e.g.) 2, 7.4] that the poset of ideals of $\Lambda$ is isomorphic to the poset of ring-theoretic ideals in the ring $\operatorname{End}(\Lambda, \Lambda)$. (We leave for the future a more concrete description of the ideals in a projective generator.)

\section{ACKNowledgements}

I am grateful to Peter Jorgensen and Paul Smith for useful remarks on an earlier draft of this note. I am also grateful for the suggestions of the referee, which helped to more clearly present the issues raised in this note.

\section{REFERENCES}

1. C. J. Pappacena, The injective spectrum of a noncommutative space, J. Algebra 250 (2002), 559-602. MR.1899866 (2003d:16007)

2. N. Popescu, Abelian categories with Applications to rings and modules, L.M.S. Monographs, Academic Press, London, 1973. MR0340375 (49:5130)

3. A. L. Rosenberg, Noncommutative algebraic geometry and representations of quantized algebras, Mathematics and its Applications, vol. 330, Kluwer, Dordecht, 1995. MR1347919 (97b:14004) 
4. S. P. Smith, Subspaces of non-commutative spaces, Trans. Amer. Math. Soc. 354 (2002), 2131-2171. MR1885647 (2003f:14002)

5. _ Maps between non-commutative spaces, Trans. Amer. Math. Soc. 356 (2004), 29272944. MR2052602 (2005f:14004)

6. J. T. Stafford and M. Van den Bergh, Noncommutative curves and noncommutative surfaces, Bull. Amer. Math. Soc. 38 (2001), 171-216. MR1816070 (2002d:16036)

7. B. Stenström, Rings of Quotients, Die Grundlehren der mathematischen Wissenschaften in Einzeldarstellungen, vol. 217, Springer-Verlag, New York, 1975. MR0389953 (52:10782)

8. M. Van den Bergh, Blowing up of non-commutative smooth surfaces, Mem. Amer. Math. Soc. 154 (2001). MR1846352 (2002k:16057)

Department of Mathematics, Temple University, Philadelphia, Pennsylvania 19122

E-mail address: letzter@math.temple.edu 